\title{
BMJ Global Health User assessments and the use of information from MomConnect, a mobile phone text-based information service, by pregnant women and new mothers in South Africa
}

\author{
Donald Skinner, ${ }^{1,2}$ Peter Delobelle, ${ }^{3}$ Michele Pappin, ${ }^{4}$ Desiree Pieterse, ${ }^{1,5}$ \\ Tonya Marianne Esterhuizen, ${ }^{6}$ Peter Barron, ${ }^{7,8}$ Lilian Dudley ${ }^{4}$
}

To cite: Skinner D, Delobelle $P$, Pappin M, et al. User assessments and the use of information from MomConnect, a mobile phone text-based information service, by pregnant women and new mothers in South Africa. BMJ Glob Health 2018;3:e000561. doi:10.1136/ bmjgh-2017-000561

Handling editor Seye Abimbola

Received 11 September 2017 Revised 29 November 2017 Accepted 9 December 2017
Check for updates

For numbered affiliations see end of article.

Correspondence to Dr Donald Skinner, Human Sciences Research Council; dskinner@hsrc.ac.za

\section{ABSTRACT}

MomConnect was designed to provide crucial health information to mothers during pregnancy and in the early years of child rearing in South Africa. The design drew on the success of the Mobile Alliance for Maternal Action's programme in South Africa, as well as a growing list of mobile health (mHealth) interventions implemented internationally. Services such as MomConnect are dependent on user acceptability as all engagements are voluntary, meaning that tools have to be easy to use and useful to be successful. This paper describes the evaluation of the tool by pregnant women and new mothers using the tool. A purposive sample of 32 individual semistructured interviews and 7 focus groups were conducted, across five provinces in South Africa. All the sessions were transcribed and then analysed using a contextualised interpretative approach, with the assistance of Atlas.ti. The women were consistently positive about MomConnect, attaching high value to the content of the messages and the medium in which they were delivered. The system was found to work well, with minor problems in some language translations. Respondents were enthusiastic about the messages, stating that the information was of great use and made them feel empowered in their role as a mother, with some saving the messages to use as a resource or to share with others. The most significant problems related to network coverage. There was strong support for this intervention to continue. Given the user acceptability of mHealth interventions, MomConnect appeared to meet the target of identifying and responding to the recipient's needs.

\section{INTRODUCTION}

The impact of mobile health (mHealth) has been well-researched in the developed world, but much less evaluation has been done in resource-limited settings. While mHealth can cover a wide range of interventions, the focus here will be on the use of text messaging. In resource-limited settings, specific contributions include the potential

\section{Summary box}

Mobile health has been found to make positive contributions to health in multiple settings, including maternal health.

- Women were consistently positive about MomConnect, attaching high value to the content of the messages and the medium in which they were delivered.

- The system was found to work well, with only mino problems in some language translations. The most significant problems related to network coverage.

- Respondents were enthusiastic about the messages, stating that the information was of great use and made them feel empowered in their role as a mother, with some saving the messages to use as a resource or to share with others.

- There was strong support for this intervention to continue and even to grow where possible.

for task shifting, better use of resources and cost savings. ${ }^{12}$ Randomised trials evaluating mHealth, including the use of text message, have shown the ability of mHealth to address a range of health challenges, including maternal and child health, ${ }^{3-5}$ and have included trials in resource-limited countries. ${ }^{6-9}$ The set-up and maintenance costs can limit the affordability of mHealth and make evaluation essential, ${ }^{10}{ }^{11}$ including user acceptability and support, which requires a different approach to looking at outcomes. ${ }^{12} 13$ Text-based interventions are dependent on user acceptability as all engagements by recipients are voluntary. For the tool to be used and to have an impact, it first has to be acceptable and usable by the target group, pregnant women and mothers.

A detailed development process for both the systems used in the interventions and the content, taking the target population into 
account and a relevant theoretical base, is crucial to the success of mHealth interventions, whether looking at measurable impacts or user acceptability. ${ }^{1}$ Some concerns have been reported in a systematic review, indicating that mHealth interventions in the public health field are often not developed in a systematic manner. ${ }^{14}$ The gaps in mHealth intervention development stem from the limited scale and scope of mHealth implementation and evaluation, ${ }^{15}$ and the current mHealth evidence base comprises mostly evidence from the field of computer science and not health, which is not sufficient to inform and influence stakeholders to invest resources in nationally scaled mHealth initiatives. ${ }^{2}{ }^{16-18}$ For these tools to transition to being directly useful, the tools need high approval and acceptance from potential recipients.

\section{MOMCONNECT}

MomConnect is a text service provided to pregnant women and new mothers. From the date that the pregnant woman registers her pregnancy at the clinic, she begins to receive messages in the language of her choice. There are standardly one to three messages a week, depending on the stage of the pregnancy. The messages continue until the child is 1 year old. It was expected that MomConnect would provide a valuable service to new mothers when registering their pregnancy at the local clinic, complementing the current set of services in empowering mothers to take care of their children. MomConnect was modelled on a similar texting intervention in South Africa, ${ }^{19}$ whereby text messages covering broad areas of child care and health were sent to pregnant mothers from the time they presented at the clinic. ${ }^{18}$

The content of the messages including examples is provided in table 1.

The registration is done at the clinic, and if no problems occur this can be done quickly. There is no cost to the mother. If the mother does not have her own phone, she can have the messages sent to another phone where she can read them. The service should be provided at all public health clinics in South Africa by health workers who have been trained, backed by public education about the service. There is an attached service, HelpDesk, that allows mothers to phone in and ask questions.

\section{EVALUATION}

The objective of this user evaluation was to assess the felt value of MomConnect, to describe the experiences of pregnant women and new mothers with MomConnect, and to obtain suggestions for its further improvement. It formed part of a larger evaluation of the programme. The qualitative research methodology used comprised both focus group discussions and semistructured indepth interviews. The focus groups and interviews lasted about 20-45 min and were digitally recorded.

Participants were purposively selected from 15 facilities in five provinces-Western Cape, KwaZulu-Natal, Free State, Gauteng and Mpumalanga-which were purposively selected to represent different language and cultural groups in South Africa. The final sample included 32 individual interviews and 7 focus groups. Within each province, three facilities were purposively selected among those serving large urban communities and those serving semirural communities or villages. Pregnant women and mothers of babies were approached while in line for services to participate. Only women who had registered on MomConnect were included. If they agreed they could either participate immediately while their place in the queue was maintained, or once they had completed their appointments. Focus groups were difficult to organise among mothers due to time limitations and queues for services. These groups were small, ranging from two to four participants, which did impact on the quality of information obtained.

The discussions, guided by a schedule, focused on the context in which women lived; the clinics; perceptions of the MomConnect system and the messages received; the registration process; and the impact of the messages on pregnant women and mothers, their children and family. Seven fieldworkers, all of whom had postgraduate qualifications, shared the tasks of doing interviews and focus

\section{Table 1 Examples of messages}

Advice on diet and to avoid alcohol

Warning signs that indicate to the mother that she should present at the clinic

Information on the development of the fetus or the child once born

Reminders to go to the clinic at different stages of the pregnancy and once the child is born

Suggestions about preparations or activities that will make it easier for the mother

Support around social or context issues
Smoking, illegal drugs and alcohol are all dangerous for you and your baby. Now is a good time to give up if you can. Ask for help at the clinic.

Spotting or light bleeding is worrying but common in pregnancy. If you have heavy bleeding or bleeding with pain, contact your clinic immediately.

Your baby's heart and brain are forming already. Take the pills from the clinic each day to help your baby grow well. They are free!

Go to the clinic for a check-up. Clinic staff will weigh your baby and test her for HIV if necessary. If you know early on, your baby can get the best care.

Get the things you will need for the birth - sanitary pads, pyjamas and wash things. Put them in a bag so you can go straight to the hospital when labour starts.

It's not OK if your partner or anyone hits or yells at you. You have the right to seek help. Talk to a friend or a health worker for advice. 
groups. All fieldworkers were trained in fieldwork over 3 days by the first author.

All interviews and focus groups were transcribed and where necessary translated by the fieldworkers themselves. The first author read 20 interviews to deepen understanding of the material to draw out themes, which were combined with the thematic areas from the objectives to establish the theme list used to code the data. A contextualised interpretative analytical approach was used, ${ }^{20-22}$ supported by Atlas.ti to facilitate analysis. The interpretations below were validated by rereading interviews after completing analysis to search for contradictory statements. The first author is a social scientist with extensive experience in public health and qualitative research, including research, teaching and student supervision. Other team members reviewed the list of themes to assess if they could discern gaps, with some other authors reading some or most of the interviews. Quotes from respondents show the province where the interview was done, and whether they were pregnant or the baby had been born. The following is the key: Free State (FS), Gauteng (GP), KwaZulu-Natal (KZN), Mpumalanga (MP) and Western Cape (WC); pregnant (P) and mother $(\mathrm{M})$.

All women interviewed gave full informed consent. The interviewees were informed that they could stop the interview at any point. All interviews were completed without incident. To protect confidentiality all identifying information was removed from the interview transcripts. The information was stored on password-protected computers. Fieldworkers returned all documents and files to the research office, and once these were secured all other copies were deleted.

While this study was larger than most qualitative studies and covered a large sector of programme implementation, the sample cannot be considered fully representative. The purposive sampling however tried to ensure that as broad a representation as possible was obtained. The interviews with women were only done with those on MomConnect, excluding those who had not connected or who had opted out. These interviews were shorter than the average for a qualitative interview as respondents were not used to this kind of self-reflection and there was insufficient time to develop a conducive relationship ahead of the interview. Women had to be drawn out of service queues for the interviews and were concerned about losing their position in the queue, despite assurances from the interviewers.

\section{CONTEXT}

Communities were commonly described by the respondents as poor, with high unemployment and poor housing. From the respondents' descriptions, the rural communities were generally worse off and had less access to transport. Grants were of considerable assistance, but were difficult to access. Safety was a general concern reported by the women, with multiple reports of crime, violence and abusive relationships. Foreigners and refugees using the clinics were particularly vulnerable, often severely impoverished and living a transient existence.

Mothers generally praised the efforts of the health workers, seeing them as courteous and caring. Respondents noted the staff worked hard to provide services, and if they were prepared to wait, everybody was seen and treated. The major complaint centred on waiting times. While some patients were done in an hour, most felt that they waited for most of the day. The respondents recognised that clinics were short-staffed, which increased waiting times and accentuated staff frustrations. The first visit to the clinic for a pregnancy was especially long, with multiple tests and counselling processes, leading to women feeling overwhelmed, with much required knowledge not being internalised. This applied particularly to first pregnancies or women dealing with a recent HIV diagnosis.

They do take care of us because I'd never lie. Even in the morning when they arrive, they show that they care about us. I never seen any of them not greeting us. (KZN P)

\section{MOTHERS' EVALUATION OF MOMCONNECT}

The pregnant women and mothers uniformly were highly appreciative of MomConnect. They reported feeling empowered and better able to manage their pregnancy and child rearing, and spoke directly about the assistance that they felt the system had provided in terms of their own health and that of their babies. The women wanted to receive the information and missed it on the few occasions when they lost contact with the service. Many actually wanted the service extended.

They (MomConnect messages) are so helpful because even after you give birth, they also tell you how to take care of the baby; in case you notice something wrong with the baby, go to the clinic. (KZN M)

A recurrent theme was the importance of informing others and that all pregnant women should receive the messages. Some women did direct advocacy themselves, sharing messages and talking about MomConnect, even registering their friends. There was a general call for MomConnect to be broadly advertised. There were no reported substantial constraints to use the programme other than access to mobile phones.

I'd like to encourage (the) MomConnect program, it needs to go further because it will help many more people. (KZN M)

A key theme was trust in and loyalty to the MomConnect messages, acknowledging this information above other advice. One woman specifically identified phone messaging as being key to getting her attention. She spoke about not even noticing or reading posters anymore. The role of mobile phones as a key communication channel appeared to be shared. 
I do not want to lie I don't read posters, I don't get interested in reading and learning about what is in the posters. (WC P)

Among the women interviewed the range of South African languages and the quality of the translations were generally felt as appropriate: "The messages are clear." However, Xhosa language messages presented concerns, with some Xhosa women requesting to receive messages in English, stating the Xhosa used was too "deep" or too traditional so it was difficult to understand. It appeared that different dialects were spoken between the Western and Eastern Cape. Respondents reported that not all languages were available in every community, and participants then had to select English.

Xhosa has deep words that are difficult to understand... Yes English at least you can even look up (English) in your dictionary. (WC M)

A small group of respondents wanted to change the language of the messages, having chosen or being assigned the wrong language at registration, but this was not possible. One woman registered again in the new language, and now received two sets of messages. Having a function that allows women to change the language of the messages that they receive would at least give them some flexibility.

No, it was just when I connected I accidentally put in Afrikaans but then I reconnected again in English...Both messages still come onto my phone but I only read the English ones because I don't understand Afrikaans. (GP M)

\section{THE MOMCONNECT SYSTEM}

The frequency and delivery time of the messages were also generally appreciated. Nobody stated there were too many messages. There were a few spontaneous requests for more messages to extend their knowledge. Respondents appeared uncertain about how long the messages would continue after birth. Instances were reported where messages arrived late at night due to problems with access to the signal.

Sometimes you get it in the morning, but usually you get it around 9:00 or 10:00...Yes, but sometimes it delays and you receive it late...And sometimes when there is a delay, they often send a message apologizing. (KZN M)

The process of registration was remembered as being easy to do and quick, with the mothers being given instructions and doing self-registration. These women felt empowered to register others or to reregister themselves if problems occurred. Alternatively nursing staff assisted and registered the women, which was also generally quick.

So I even showed a friend of mine who is pregnant and told her that she can dial this number because she is not connected on MomConnect... So I showed her how to do it from the paper. (WC P)
There were occasional problems at the time of registration due to network access. At clinics where the signal strength was poor, the health workers often took the women's details and registered them later where the signal was better. These problems caused delays and increased waiting times.

We dialled that star-something, but the network that day was not good. (KZN P)

A major concern for HIV-positive women was the risk that the messages may reveal their status. One HIV-positive woman concerned that her mother-in-law might find out her status kept the messages hidden until she felt reassured her status would remain confidential. Then she began sharing the information she obtained. By the time of the interviews, all HIV-positive women in the sample were confident in the confidentiality of MomConnect. Given that only women who had registered with MomConnect were interviewed, we could not assess if this fear led to women not registering.

I asked her to select English because some people have access to my phone, people fiddle with phones and read the messages. (WC M)

\section{ROLE AND FELT IMPACT OF MOMCONNECT}

The women, especially first-time mothers, felt that they had a lot to learn and drew support and confidence from the messages. All the messages were appreciated. The pregnant women emphasised the messages on the types of food to eat and to avoid; reminders of clinic visits; health problems to be aware of; what pills and health additives to take; and interaction with the fetus. Among those with babies, they also emphasised information on food, breast feeding, immunisation, medications, symptoms and clinic visits, and how to bond with the child. The efforts to test the messages used in MomConnect in advance and the knowledge of those who composed the messages appeared to contribute to the success. ${ }^{23}$ This also meant MomConnect met the requirement of being responsive to the target group's needs. ${ }^{1} 17$

When I registered with MomConnect they informed me that 'I must pack my ID, my clothes, sanitary towels and the child's clothes' things like that...It was simple to raise a child with MomConnect because I took every advice I got from MomConnect. (MP P)

Women expressed particular excitement about information relating to the development of the fetus as this increased their relationship with the unborn baby. Women spoke of feeling that they could visualise the baby's development, leading them to take care and be more aware of health concerns. They now felt they "(were) actually carrying a living person," which made them happy. They felt better prepared and had greater understanding of what was going on, both during pregnancy and after the birth. Mothers with previous children did not claim as much benefit as they could reflect on their previous 
pregnancies, but were generally still enthusiastic. They were acquiring new information, which made this pregnancy easier.

They sent me something that my unborn has eye-brows, that way I can see my baby's development. (KZN P)

The women spoke of having a greater understanding of pregnancy and child care, which allowed them to plan around expected events, to understand what was happening and to make sense of the symptoms that they were experiencing. This empowered the women to decide when they needed to visit the clinic, reducing the risk of problems escalating and of inappropriate calls.

The messages play a huge role; like, you receive a message explaining something that you are already experiencing and you were about to go to the clinic 'for no reason'. (KZN M)

A common practice was to save the messages, as a reference in case they wanted to go back at a later stage and check information, "from time to time I refer back and read." This was not universal and some read and deleted the messages. This last group tended to be older and more educated women or women who already had children. Generally, those participants who were able to, shared the messages with their families and especially the baby's father, who were reported to be excited about the new information. The messages also provided a base for discussion. Messages were also shared with close friends and colleagues. The sharing of certain messages, such as around domestic violence, left the women feeling supported. There were many suggestions for messages to be developed for fathers, who were reported to show great interest in the existing set of messages.

He can see that the message is written MomConnect, and when he reads it. For my side, if I left my phone at home, when I get back my partner tells me that I have a message from MomConnect. (FS P)

The different use between first-time mothers and those bearing a subsequent child could allow for two different sets of message intensity. The current system could be maintained for first-time mothers, while a less message intensive version could be offered to mothers who had a previous pregnancy, increasing programme affordability. This should remain a choice for the mothers.

\section{CRITICISM}

Despite being directly asked, no respondents stated that any messages were patronising or offensive. Even the messages on HIV were found to be non-offensive and non-discriminatory. Some terms such as 'high-high', referring to high blood pressure, were not understandable to all. A message stating the baby should move five or six times every day raised concern, as some women claimed they could not feel this. The clinic nurses were able to clarify their concerns.
It is clear to me although I got confused when I received the message about what to do when the baby is not moving at all...I asked the nurse because that got me really scared but she managed to make me understand. (FS P)

There was very poor awareness of the HelpDesk. The service was only raised by a few women, but there was an expressed need for the service. The few who had used the service felt it to be useful. Concerns were raised about the cost of using the service.

There was a time when my child couldn't pass the stools for two days. I was at work and I called them. They told me that because she is still being breastfed. (MP M)

\section{CONCLUSION}

Respondents were extremely positive about MomConnect and its role in assisting their pregnancy, their health and that of their children. The system was felt to be good in that messages were delivered as expected and most women were easily registered. The service was free and having the messages delivered by a mobile phone appeared to increase credibility and attention. Problems with mobile phone reception need to be addressed both in terms of registration and message delivery. There was strong support for the programme to be continued and extended. This was shown in the confidence that the respondents expressed as a result of the knowledge, the reported improved health behaviours and the excitement that they felt about their role. This is similar to the results found in other studies such as the Text4Baby programme. ${ }^{24}$ This excitement was often shared by other family members, especially the fathers of the children. There are areas where MomConnect does need to improve, including problems with registration and some language issues. This approach can also provide direction on how to approach the use of text messaging tools in other contexts. Of particular importance here is the felt value of the messages for the recipients. The responses indicate that the mothers developed a particular connection with the messages, plus there was good trust in the source. This may be key to getting positive user support for such interventions.

\section{Author affiliations}

${ }^{1}$ Research on Health and Society Unit, Department of Global Health, Faculty of Medicine and Health Sciences, Stellenbosch University, Stellenbosch, South Africa ${ }^{2}$ HIV, AIDS, STD and TB, Human Sciences Research Council, Pretoria, South Africa ${ }^{3}$ School of Public Health, University of the Western Cape, Cape Town, South Africa ${ }^{4}$ Community Health Division, Department of Global Health, Faculty of Medicine and Health Sciences, Stellenbosch University, Stellenbosch, South Africa

${ }^{5}$ Research Unit, Ndlovu Care Group, Groblersdal, Limpopo, South Africa

${ }^{6}$ Division of Epidemiology and Biostatistics, Faculty of Medicine and Health Sciences, Stellenbosch University, Stellenbosch, South Africa

${ }^{7}$ HIV/AIDS, TB and MCWH, National Department of Health, Pretoria, Gauteng, South Africa

${ }^{8}$ School of Public Health, University of the Witwatersrand, Johannesburg, South Africa

Acknowledgements In addition to the financial support, we would like to thank the staff of CDC South Africa for their input and advice at different stages of the project. We would like to thank the National Department of Health for assisting us with access to health facilities and for their input and advice on the project. We would like to thank all the fieldwork staff who assisted in collecting the data. 
Finally, we would like to thank the staff of the clinics and the patients who assisted us and participated in the study. Without all of this assistance, the project would not have been possible. The support provided by John Snow, Inc. (JSI) in the President's Emergency Plan for AIDS Relief (PEPFAR) and United States Agency for International Development (USAID)-funded MEASURE Evaluation Strategic Information for South Africa (MEval-SIFSA) project to enable this publication is acknowledged with gratitude.

Contributors The first author led the design and analysis of the paper. All the other authors contributed to the design of the methodology, assisted in the oversight of the fieldwork, contributed to the development of themes, read and did validity checks on the earlier reports on the data, and read and verified the current content of the paper.

Funding This project has been supported by the US President's Emergency Plan for AIDS Relief (PEPFAR) through the US Centers for Disease Control and Prevention (CDC) under the terms of cooperative agreement (U2GGH000460).

Competing interests None declared.

Patient consent Obtained.

Ethics approval The full protocol for this study received ethical approval from the Stellenbosch University Health Research Ethics Committee.

Provenance and peer review Not commissioned; externally peer reviewed.

Data sharing statement There are currently some unpublished data that the authors will be working on to publish further. No formal plans have been made for the data to be curated for sharing.

Open Access This is an Open Access article distributed in accordance with the Creative Commons Attribution Non Commercial (CC BY-NC 4.0) license, which permits others to distribute, remix, adapt, build upon this work non-commercially, and license their derivative works on different terms, provided the original work is properly cited and the use is non-commercial. See: http://creativecommons.org/ licenses/by-nc/4.0/

(C) Article author(s) (or their employer(s) unless otherwise stated in the text of the article) 2018. All rights reserved. No commercial use is permitted unless otherwise expressly granted.

\section{REFERENCES}

1. Toefy Y, Skinner D, Thomsen S. "Please don't send us spam!" a participative, theory-based methodology for developing an mhealth intervention. JMIR Mhealth Uhealth 2016;4:e100.

2. Tomlinson M, Rotheram-Borus MJ, Swartz L, et al. Scaling up mHealth: where is the evidence? PLoS Med 2013;10:e1001382.

3. Aranda-Jan CB, Mohutsiwa-Dibe N, Loukanova S. Systematic review on what works, what does not work and why of implementation of mobile health (mHealth) projects in Africa. BMC Public Health 2014;14:188.

4. Tamrat T, Kachnowski S. Special delivery: an analysis of mHealth in maternal and newborn health programs and their outcomes around the world. Matern Child Health J 2012;16:1092-101.

5. Gazmararian JA, Elon L, Yang B, et al. Text4baby program: an opportunity to reach underserved pregnant and postpartum women? Matern Child Health J 2014;18:223-32.
6. Cole-Lewis H, Kershaw T. Text messaging as a tool for behavior change in disease prevention and management. Epidemiol Rev 2010;32:56-69.

7. Krishna S, Boren SA, Balas EA. Healthcare via cell phones: a systematic review. Telemed J E Health 2009;15:231-40.

8. Vervloet M, Linn AJ, van Weert JC, et al. The effectiveness of interventions using electronic reminders to improve adherence to chronic medication: a systematic review of the literature. J Am Med Inform Assoc 2012;19:696-704.

9. Horvath T, Azman H, Kennedy GE, et al. Mobile phone text messaging for promoting adherence to antiretroviral therapy in patients with HIV infection. Cochrane Database Syst Rev 2012;3:CD009756.

10. Broomhead S, Jones T. eHealth economics and finance for Africa: a white paper. South Africa: tinTree International eHealth, 2011.

11. Free C, Phillips G, Felix L, et al. The effectiveness of M-health technologies for improving health and health services: a systematic review protocol. BMC Res Notes 2010;3:250.

12. Kushniruk AW, Borycki EM, Kuwata S, et al. Emerging approaches to usability evaluation of health information systems: towards in-situ analysis of complex healthcare systems and environments. Stud Health Technol Inform 2011;169:915-9.

13. Yardley L, Morrison LG, Andreou $P$, et al. Understanding reactions to an internet-delivered health-care intervention: accommodating user preferences for information provision. BMC Med Inform Decis Mak 2010;10:52

14. Whittaker R, McRobbie H, Bullen C, et al. Mobile phone-based interventions for smoking cessation. Cochrane Database Syst Rev 2016:4:CD006611.

15. Maar MA, Yeates K, Toth Z, et al. Unpacking the black box: a formative research approach to the development of theory-driven, evidence-based, and culturally safe text messages in mobile health interventions. JMIR Mhealth Uhealth 2016;4:e10.

16. Marshall C, Lewis D, Whittaker M. mHealth technologies in developing countries: a feasibility assessment and a proposed framework. 50: Health Information Systems Knowledge Hub, University of Queensland, 2013.

17. Chang LW, Njie-Carr V, Kalenge S, et al. Perceptions and acceptability of mHealth interventions for improving patient care at a community-based HIV/AIDS clinic in Uganda: a mixed methods study. AIDS Care 2013;25:874-80.

18. MAMA. Healthy pregnancy, healthy baby text messaging service. Tanzania: MAMA: Mobile Alliance for Maternal Action, 2013.

19. MAMA, Mobile Alliance for Maternal Action. Global monitoring \& evaluation framework. Washington: Maternal and Child Health Integrated Programme, USAID, 2012.

20. Skinner D. Qualitative research methodology: an introduction. In: Ehrlich R, Joubert G, eds. Epidemiology: A research manual for South Africa. 3rd edn. Cape Town: Oxford University Press, 2014

21. Terre Blanche M, Durrheim K, Kelly K. First steps in qualitative data analysis. In: Terre Blanche M, Durrheim K, Painter D, eds. Research in Practice: Applied Methods for the Social Sciences. 2nd edn. Cape Town: UCT Press, 2006.

22. Kelly K. Lived experience and interpretation: the balancing act in qualitative analysis. In: Terre Blanche M, Durrheim K, Painter D, eds. Research in practice: applied methods for the social sciences. 2nd edn. Cape Town: UCT Press, 2006.

23. Coleman J. Monitoring MAMA: gauging the impact of MAMA South Africa. J Mob Technol Med 2013;2:9.

24. Evans WD, Wallace JL, Snider J. Pilot evaluation of the text4baby mobile health program. BMC Public Health 2012;12:1031. 